\title{
Research progress of analysis method of slope stability combined with hot spots
}

\author{
XIE Qiao $^{1, *}$, ZHANG Jia-sheng ${ }^{1}$, and Wang Xuan ${ }^{1}$ \\ ${ }^{1}$ School of Civil Engineering, Central South University, Changsha, Hunan 410075, China
}

\begin{abstract}
The analysis methods and theories of slope stability are constantly improving and developing, and more methods in line with the development characteristics of the times have been put forward by scholars at home and abroad. Combined with the latest research hot spots in the direction of analysis methods of slope stability in the world, the analysis methods of slope stability are systematically classified and summarized; The principles, characteristics, scope of application of various methods are summarized; The development trend of slope stability analysis is comprehensively analyzed from various aspects. It provides the follow-up scholars with the research of new methods. It can provide reference for the selection of evaluation methods of practical slope engineering stability.
\end{abstract}

\section{Introduction}

According to the common habits of classification, these traditional methods can be roughly divided into qualitative analysis methods, quantitative analysis methods and uncertainty analysis methods[1]. With the rapid development of science and technology, the theory and method of slope stability analysis have been constantly developed and improved. Based on the latest research hot spot of the analysis methods of slope stability of international researchers [2], we adds machine learning methods on the basis of traditional classification.

\section{Methods of slope stability analysis}

\subsection{Qualitative analysis methods}

The qualitative analysis method is mainly based on the relevant geological data obtained from field geological survey to analyze the influencing factors of slope stability, the possible failure forms and the mechanical mechanism of instability deformation and failure. The advantage of qualitative analysis method is that it comprehensively considers all kinds of factors affecting the slope stability, and can quickly get the preliminary judgment of slope stability; the disadvantage is that it mainly depends on experience, and can only qualitatively analyze the development trend of slope stability to be studied. At present, the main classification of qualitative analysis methods commonly used in the stability analysis and evaluation of practical slope engineering in the world is shown in Figure 1.

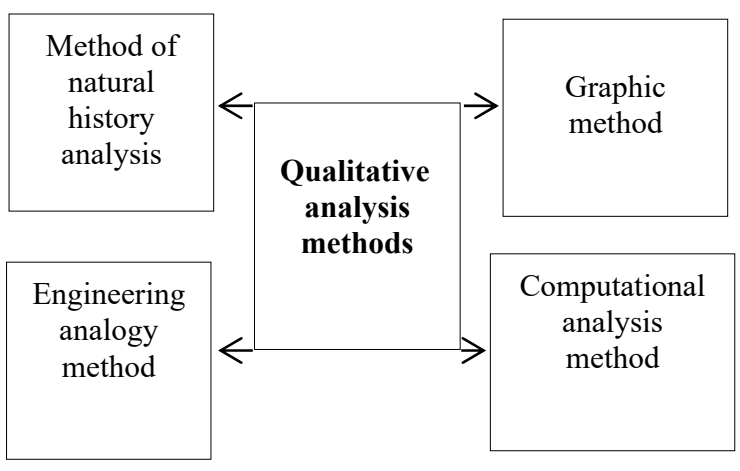

Fig. 1. Qualitative analysis methods.

\subsection{Quantitative analysis methods}

The quantitative analysis method is mainly based on the theories and methods of rock mechanics, soil mechanics and theoretical mechanics, combined with advanced computer technology, to obtain the mechanical deformation mechanism of landslide mass, to solve the safety factor of slope stability evaluation, so as to make a more quantitative evaluation of slope stability. Strictly speaking, at present, the slope stability analysis has not fully entered the determination. The quantitative stage can only be regarded as a semi quantitative stage. The main classification of quantitative analysis methods commonly used in the stability analysis and evaluation of practical slope engineering at home and abroad is shown in Fig. 2. Each method has its own outstanding characteristics and shortcomings.

\footnotetext{
* Corresponding author: xie_qiao@csu.edu.cn
} 


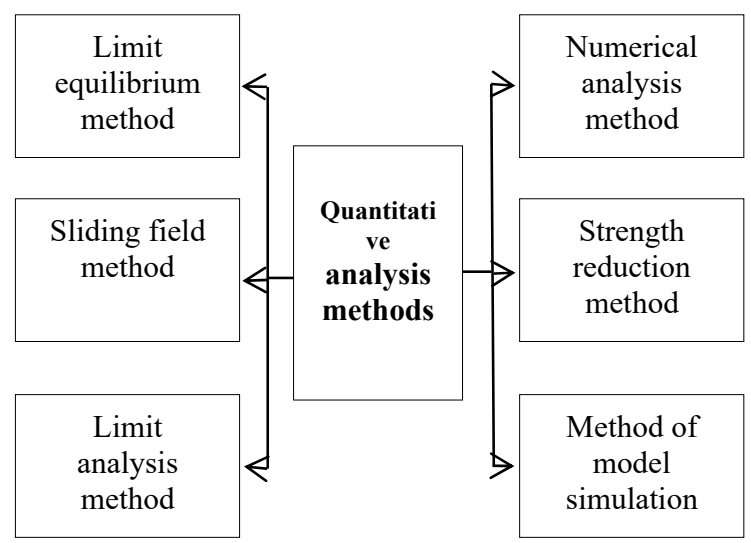

Fig. 2. Quantitative analysis methods.

The limit equilibrium method is mainly to determine the potential most dangerous sliding surface of the slope by calculating the safety factor and critical failure load of the slope according to the static equilibrium condition of the landslide mass on the known slip surface. It is necessary to know the distribution and shape of slip surface. When the shape of slip surface is not simple plane, the slice method is usually used to solve this kind of statically indeterminate problem. According to the different assumptions and equations, it can be divided into non strict slice method and strict slice method. The parts of various slice methods put forward by scholars from various countries are shown in Fig. 2. Based on the two-dimensional analysis method, many scholars have begun to study the three-dimensional limit equilibrium method [3-5]. The results of the block limit equilibrium method mainly reflect the real stress-strain relationship of rock and soil. The stability parameters obtained are the average values of each sliding block on the sliding surface, which are assumed, and also reflect the deformation and failure mechanism of slope in the critical state of deformation and failure. It is impossible to analyze the complete process of the occurrence and development of slope deformation and failure, and it is difficult to accurately consider progressive failure The influence of bad on slope stability. However, this method is simple, intuitive, easy to understand and mature in theory. It is suitable for the study of dynamic change of water pressure and discontinuous rock mass and soil mass. It is a traditional method used to analyze slope stability in engineering circles at home and abroad.

\subsection{Uncertainty analysis methods}

The uncertainty analysis method is mainly due to the complex geological conditions of the slope structure, and has greater uncertainty and unknown characteristics, so some uncertainty methods are introduced to analyze and evaluate the stability of the slope. At present, this method has achieved certain results in the aspect of slope stability evaluation. However, the method itself is more dependent on the quantity of relevant geological data that can be obtained by the slope structure to be evaluated. Therefore, the accuracy of the results may not be strong and the mathematical derivation process of some methods is more complex when there is no data. The main classification of uncertainty analysis methods is shown in Figure 3.

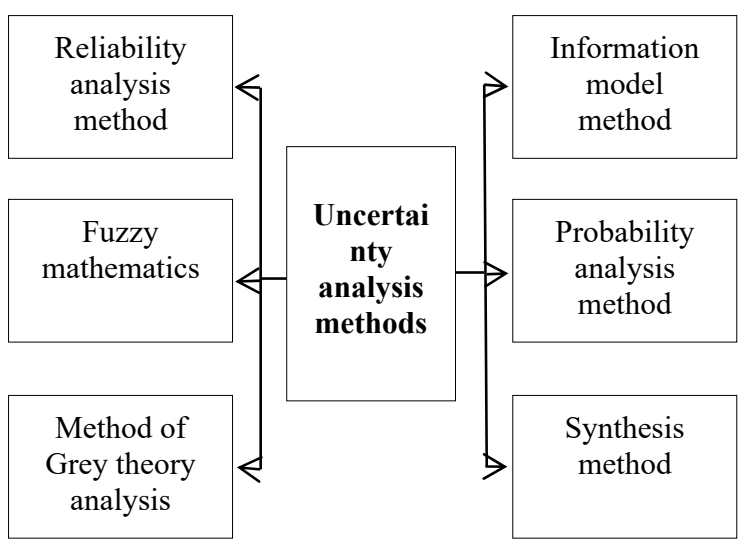

Fig. 3. Uncertainty analysis methods.

\subsection{Machine learning methods}

The relationship between the stability of the slope structure and various factors affecting the stability of the slope is a very complex nonlinear relationship, and there are many factors affecting the slope stability. Therefore, it is difficult to find a relationship that can reflect the nonlinear mapping relationship [6]. However, with the rapid development of machine learning technology in various industries in recent years, geotechnical engineering researchers seem to find that it is a good idea to introduce machine learning algorithm into slope stability analysis. Machine learning algorithm can be used to quickly study the complex nonlinear between the slope stability (stability evaluation index) and various influencing factors Relationship. At present, the method is still in continuous development and improvement, and various algorithms are constantly introduced and improved by scholars at home and abroad to improve the accuracy and reliability of the calculation results, so the method has a large space for development. The classification method of this paper is shown in Figure 4.

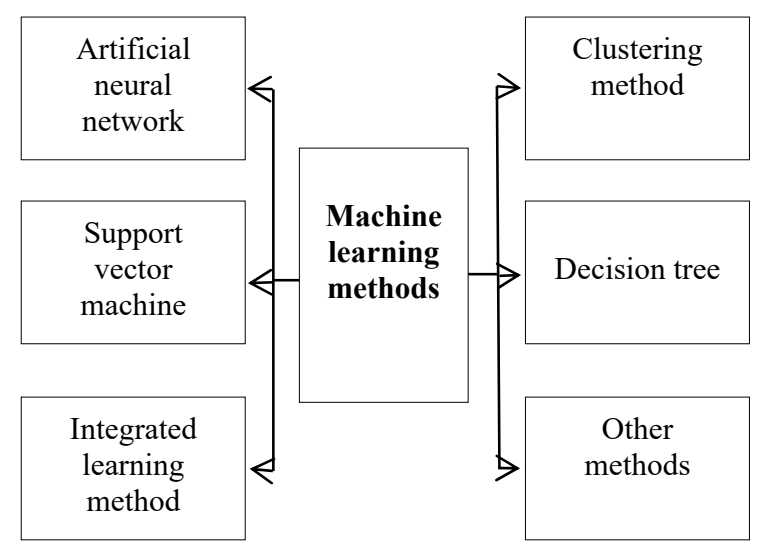

Fig. 4. Machine learning methods. 
Artificial neural network mainly refers to biological neural system, and simulates some functional characteristics of human brain by means of abstraction, simplification and simulation. This method has high fault tolerance, self-learning, self-organization, high nonlinearity, dynamic processing, associative memory and other functions. This method is introduced into slope stability analysis to overcome the shortcomings of traditional BP network, which are easy to fall into local optimum and slow convergence. Domestic and foreign scholars introduce various algorithms to make up for the shortcomings of BP network and improve the method [79]. Or the neural network algorithm is combined with other methods to obtain higher accuracy of the model [10-14]. The continuous development and progress of artificial neural network has opened up a new way and provided new ideas for the analysis of complex slope stability problems.

\section{Prospect of slope stability analysis and evaluation}

In practical engineering, the instability of slope may lead to the loss of social economy and even life safety. Therefore, in the actual project construction, it is important to analyze and evaluate the slope stability. The problem of slope stability analysis and evaluation is always a classic and full of discussion. With the continuous progress of research methods and technologies in the world, slope stability analysis has initially entered the stage of system engineering analysis and research. New theories and methods have been gradually applied to slope stability analysis and research, which broadens the development prospect of quantitative evaluation of slope stability in horizontal and vertical directions.

1) The cross introduction of various disciplines makes the theory and method of slope stability analysis develop in the direction of multi-dimensional innovation. The theories of mathematics, physics, computer and other disciplines are continuously introduced into slope stability analysis, which improves the accuracy of slope stability analysis results and the dimension of sustainable development of analysis methods.

2) Under the guidance of the existing slope stability analysis theory, the model test of the structure is strengthened to provide ideas for the new theory. Because of the complexity of the geological conditions of slope structure, it can provide more effective support for the results of slope stability analysis and evaluation to determine the similarity index of test model which is more suitable for the actual engineering situation and carry out the model test more in line with the actual engineering situation.

3) Continue to enrich and improve the comprehensive evaluation method of slope. Due to the uncertainty of slope engineering, the combination of various methods can provide more possibilities for improving the solution range or efficiency of slope stability analysis and evaluation, and can also combine innovative analysis methods with high fidelity and high precision to meet the engineering needs.

4) With the continuous development of social research means and innovative technology, the establishment of a more efficient and accurate intelligent analysis and evaluation system has become one of the directions of researchers. Combined with various kinds of practical engineering case database, according to the actual project site monitoring, analysis, feedback results and other information, the dynamic adjustment and more comprehensive system of intelligent analysis and evaluation of slope stability is established Price system.

\section{Conclusion}

Combined with the current international research hot spots, a more comprehensive and systematic arrangement of various slope stability evaluation methods at home and abroad, analysis of the development trend of slope stability evaluation, in order to provide inspiration and ideas for the later new methods or theories.

1) The analysis methods of slope stability are comprehensively and systematically sorted out in this paper, which are mainly divided into qualitative analysis method, quantitative analysis method, uncertainty analysis method and machine learning method.

2) The qualitative analysis method is mainly empirical analysis method, which comprehensively analyzes various factors affecting the slope stability, and makes qualitative description and evaluation on the stability development of the studied slope. The commonly used methods include natural cause (History) analysis method, engineering analogy method, graphic method and computational analysis method.

3) Among the quantitative analysis methods, limit equilibrium method and numerical analysis method are widely used in the world. The basic principle of the limit equilibrium method is that the landslide mass with known slip surface satisfies the static equilibrium condition, so as to obtain the average value of the slope safety factor of each block on the slip surface, but it is unable to analyze the complete development process of the slope deformation and failure; the numerical analysis method is mainly developed and perfected with the rapid development of computer technology, which can simulate the actual deformation of slope The development process is often used to assist other methods to deal with slope stability analysis problems with more complex geological conditions.

4) In recent years, with the continuous in-depth study of slope stability theory, it is found that there are uncertainties and unknowns in slope stability analysis. Therefore, scholars at home and abroad combine these uncertain factors to study slope stability and develop uncertainty analysis methods for slope stability evaluation.

5) The machine learning method can establish a prediction model based on the existing data to describe the complex nonlinear mapping relationship between the influencing factors of slope stability, and make up for the 
defect that qualitative analysis method, quantitative analysis method and uncertainty analysis method can not effectively and accurately evaluate the slope stability in a short time. In the case of lack of geological data and short construction period, the slope stability evaluation can be completed quickly.

6) The development trend of slope stability analysis method is proposed from the aspects of slope stability development theory and method, model test, composite method comprehensive evaluation and dynamic slope stability intelligent evaluation system.

\section{References}

1. Li, C.H., Liu T.P., et al. (2020)Research progress and Prospect of slope stability analysis methods. J. Northeast water conservancy and hydropower, 38 (02): 63-65.

2. Fan, Qi. (2019)Study on rapid evaluation method of highway rock slope stability based on whole process of machine learning. D. Chengdu University of technology.

3. Ma, G.L., Shao, Y.G. (2016)Stability study of threedimensional limit equilibrium slope based on Spencer method, J. Hydrogeology and engineering geology, (3): 118-123.

4. Peng, F.G., Chen,Y.Z., et al. (2017)Influence analysis of replacement tunnel on slope stability of Baihetan, J. Water conservancy planning and design, (08): 112-115.

5. Hungr, O. (2015)An extension of Bishop's simplified method of slope stability analysis to three dimensions, J. Geotechnique, 37(1):155-156.

6. Sun, C. (2017)Design of slope stability early warning system, D. Hunan University.

7. Gordan, B., Armaghani, D.J., Hajihassani, M., et al. (2016)Prediction of seismic slope stability through combination of particle swarm optimization and neural network, J. Engineering with Computers, 32(1):85-97.

8. Hu, J., Dong, J.H., Wang, K.K., et al. (2016)Study on cpso-bp model of slope stability, J. Geotechnical mechanics, (S1): 577-590.

9. Lu, C.J., Hu, H.T. (2016)Application of improved BP network in rock slope stability evaluation, J. Journal of rock mechanics and engineering, 18 (3): 303-307.

10. Chen, C.F., Yang, Y. (2016)T-S fuzzy inference system for slope stability evaluation driven by hgaann, J. Journal of rock mechanics and engineering, 24 (19): 3459-3464.

11. Guo, R.Q., Muhtar, Z.R., Liu, X.X. (2016)Evaluation method of rock slope stability based on adaptive neuron fuzzy inference system, J. Journal of rock mechanics and engineering, 25 (z1): 002785-2789.

12. Fu, Y.X, Liu, S.K, Liu, D.P. (2016)Prediction of slope rock mass stability based on RBF neural network, J. Journal of Wuhan University of Technology (Transportation Science and Engineering Edition), 27 (2): 170-173.

13. Xue, X.H., Zhang, A.H., Liu, H.J. (2016)Slope stability evaluation based on genetic algorithm and fuzzy neural network, J. Geotechnical mechanics, 28 (12): 2643-2648.

14. Xue, X.H., Zhang, A.H., Liu, H.J. (2016)Slope stability evaluation based on SOFM neural network, J. Geotechnical mechanics, 29 (8): 2236-2240. 